\title{
Combination gene therapy with p53 and Thoc1/p84 is more effective than either single agent in an animal model of human pancreatic adenocarcinoma
}

\author{
SHENMIN YIN ${ }^{1}$ and DAVID W. GOODRICH ${ }^{2}$ \\ ${ }^{1}$ Molecular Biology Program, Memorial Sloan-Kettering Cancer Center, New York, NY 10021; ${ }^{2}$ Department of Pharmacology \\ and Therapeutics, Roswell Park Cancer Institute, Elm and Carlton Streets, Buffalo, NY 14263, USA
}

Received October 7, 2005; Accepted November 18, 2005

\begin{abstract}
Gene therapy utilizing tumor suppressor gene p53 has been tested in a number of cancers in both preclinical models and clinical trials. Despite the ability of p53 to significantly inhibit tumor growth in preclinical models, it has met with more limited clinical success. This is due, in part, to the observation that a substantial fraction of tumors are resistant to the effects of p53 expression. We have tested whether combination therapy with Thoc1/p84 and p53 is more effective than single gene therapy in a p53-resistant tumor model of human pancreatic adenocarcinoma. Infection with Thoc1/p84 and p53 adenovirus inhibits pancreatic cancer cell growth in vitro and in vivo to a greater extent than treatment with either one alone at the same total viral dose. Similar results are observed with Thoc1/p84 and $R b 1$ gene therapy. The data support the use of therapy that targets both the p53 and Thoc1/p84 pathways in tumors that are resistant to perturbation of the p53 pathway alone.
\end{abstract}

\section{Introduction}

Cancer is a result of defects in the coordination of the cell cycle and cell survival. The extent of cell death is physiologically controlled by activation of cellular suicide pathways that result in morphologically and biochemically distinguishable forms of death, including apoptosis $(1,2)$. Since the benefits of traditional therapy often rely on triggering the apoptotic death of tumor cells, gene therapy-mediated induction of apoptosis or restoration of apoptotic sensitivity in tumors may provide potentially effective means to treat cancer in general $(3,4)$ and pancreatic adenocarcinoma in particular $(5,6)$. For example, adenoviral-mediated p53 gene therapy has been demonstrated to suppress the growth of human pancreatic

Correspondence to: Dr David W. Goodrich, Department of Pharmacology and Therapeutics, Roswell Park Cancer Institute, Elm and Carlton Streets, Buffalo, NY 14263, USA

E-mail: david.goodrich@roswellpark.org

Key words: pancreatic adenocarcinoma, apoptosis, gene therapy, retinoblastoma gene cancer cells by induction of apoptosis $(7,8)$. However, transduction of the p53 gene does not inhibit the growth or increase chemosensitivity of all pancreatic cancer cell lines that have been tested (9). Relative resistance to the effects of p53 may account for the modest success of p53 gene therapy as a single agent in clinical trials $(10,11)$. By analogy to combination chemotherapy, maximizing the effectiveness of cancer gene therapy probably requires the targeting of multiple, distinct molecular pathways that function in cell proliferation and cell survival.

The Thoc1/p84 gene has been cloned based on the ability of its encoded protein to bind an amino terminal domain of the $\mathrm{Rb} 1$ protein (12). The Thoc1/p84 protein contains sequence similarity to the death domains of other proteins involved in the regulation of apoptosis (13). Indeed, overexpression of Thoc1/p84 induces apoptosis from within the nuclei (14) of tumor cells but not normal fibroblasts (15). Thoc $1 / \mathrm{p} 84$ usually functions in the THO/TREX complex to regulate transcriptional elongation and RNA processing (16-18). Deregulation of Thoc1/p84 expression has also been correlated with clinicopathological variables, including tumor size and metastasis, in breast cancer (19). These observations suggest a rationale for using Thoc1/p84 as a gene therapy for cancer, a concept which has been validated in preclinical animal models $(15,20)$. Interestingly, inhibition of tumor cell growth by Thoc1/p84 gene therapy does not require p53 $(21,22)$. Since Thoc1/p84 and p53 inhibit tumor cell growth through distinct mechanisms, they may be good candidates for use in combination gene therapy. To test this hypothesis, we compare the effectiveness of p53 or Thoc1/p84 gene transfer alone or in combination in reducing the growth of human pancreatic adenocarcinoma in vitro and in vivo.

\section{Materials and methods}

Cell line and cell culture. All cell lines were obtained from American Type Culture Collection (ATCC) and maintained in Dulbecco's modified Eagle's medium (DMEM) with $10 \%$ FBS and antibiotics (100 unit $/ \mathrm{ml}$ penicillin, $100 \mu \mathrm{g} / \mathrm{ml}$ streptomycin) in a $5 \% \mathrm{CO}_{2}$ incubator at $37^{\circ} \mathrm{C}$.

Recombinant adenovirus. Full-length Thoc1/p84 cDNA was subcloned into the pAdCMV (AS)-BGHpa vector and used 
to generate replication defective adenovirus (AdN5) as previously described (20). The adenovirus designed to express a phosphorylation-resistant RB1 protein ( $\mathrm{Ad} \Delta \mathrm{CDK}$ ) was constructed using identical vectors and was generously provided by Dr T.J. Liu (University of Texas M.D. Anderson Cancer Center). The p53 expressing adenovirus (Adp53) was provided by the Vector Core Laboratory of the University of Texas M.D. Anderson Cancer Center. All of the resulting adenovirus were modified serotype 5, E1 deleted, with relevant gene expression under control of the cytomegalovirus early promoter. Viral stocks were propagated in 293 cells and purified by $\mathrm{CsCl}$ gradient centrifugation. The titer of concentrated stocks was determined by limiting dilution, infection of 293 cells, and determination of cytopathic effect. Viral infection was carried out in vitro by addition of the virus to DMEM medium without serum. The cells were incubated at $37^{\circ} \mathrm{C}$ for $1 \mathrm{~h}$ with constant agitation, at which point complete medium was added and cells were incubated at $37^{\circ} \mathrm{C}$ for the desired length of time.

Western blot analysis. Infected cells were lysed in a buffer containing $50 \mathrm{mM}$ Tris, pH 7.4, $250 \mathrm{mM} \mathrm{NaCl}, 5 \mathrm{mM}$ EDTA, $0.1 \% \mathrm{NP} 40,50 \mathrm{mM} \mathrm{NaF}, 1 \mathrm{mM}$ PMSF, and $1 \mu \mathrm{g} / \mathrm{ml}$ leupeptin. Samples were kept on ice for 10 min followed by centrifugation to pellet insoluble debris. Total protein concentration of the soluble extract was determined by Bradford assay according to the manufacturer's instructions (Bio-Rad). Seventeen micrograms of total soluble protein for each sample was resolved on $10 \%$ SDS-PAGE gel and the resolved proteins were transferred to nitrocellulose membrane. The membranes were blocked with $5 \%$ dry milk in phosphate-buffered saline and probed with mouse anti-human Thoc1/p84 monoclonal antibody (12). Blots were also probed using a mouse antihuman B-actin monoclonal antibody (Oncogene Research Products) as an internal protein loading control. Probed blots were stained using a horseradish peroxidase conjugated rabbit anti-mouse IgG secondary antibody and developed using chemiluminescence (Amersham).

Cell growth assay. Cells were plated at a density of $2 \times 10^{4}$ cells/ml in 24-well plates in triplicate. Cells were infected with AdN5, AdGFP or PBS as above. Cells were harvested at indicated time intervals and viable cells were counted using trypan blue and a hemacytometer.

Apoptosis assays. For FACS analysis, $72 \mathrm{~h}$ after adenovirus infection, adherent and detached cells were combined and fixed overnight with $75 \%$ ethanol in PBS at $4^{\circ} \mathrm{C}$. After rinsing two times with PBS, cells were incubated for $30 \mathrm{~min}$ with $1 \mathrm{ml}$ of PBS containing $1 \mathrm{mg}$ of boiled RNase at $37^{\circ} \mathrm{C}$. Cells were then stained in $1 \mathrm{ml}$ of PBS containing $10 \mu \mathrm{g}$ of propidium iodide and a total of $2 \times 10^{4}$ were analyzed in a flow cytometer (FACScalibur; Becton-Dickinson).

Inhibition of tumor growth in vivo. One million FG cells in a volume of $0.1 \mathrm{ml}$ PBS were injected subcutaneously into the flanks of nude mice. Once tumor volumes reached $40-50 \mathrm{~mm}^{3}$, $100 \mu \mathrm{l}$ of adenovirus suspension $\left(1.0 \times 10^{7} \mathrm{pfu}\right)$ was injected intratumorally weekly for 3 weeks. PBS was used as a vehicle for sham control. Tumor formation was evaluated weekly for
A

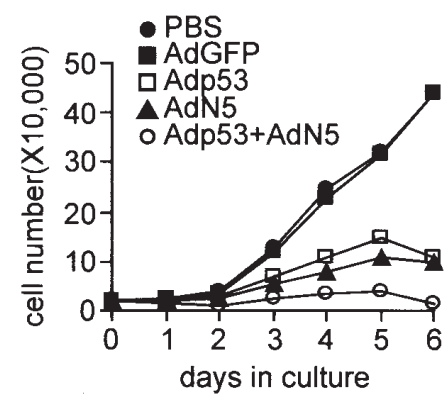

B

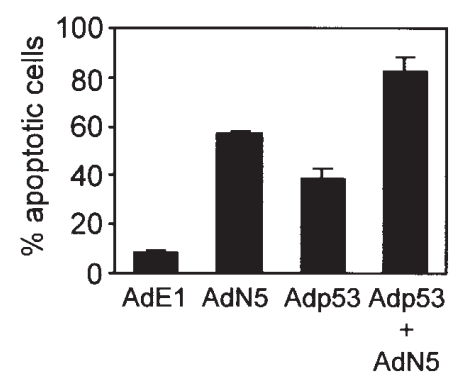

Figure 1. Effects of combination Thoc1/p84 and p53 gene therapy on pancreatic cancer cells in vitro. A, an equal number of FG cells were plated and treated at an MOI of 50 with the indicated virus or PBS. The total MOI of the combination of AdN5 and Adp53 (1:1) was 50. Aliquots of the infected cells were harvested at the indicated times and the number of cells excluding trypan blue were counted using a hemacytometer. The data presented are the mean of three infections. The standard deviations from the mean are smaller than the symbols representing the data points. B, FG cells were infected with the indicated adenovirus at an MOI of 50 and harvested $72 \mathrm{~h}$ later. The percentage of apoptotic cells was measured by Annexin V staining and flow cytometry. The data represent the mean of three independent experiments.

6 weeks. At least six mice were injected for each sample. Tumor volumes were estimated by measuring the diameter of the resulting tumors in three dimensions.

\section{Results}

The cell line used to study the effects of AdN5 (Thoc1/p84 adenovirus) and Adp53 (p53 adenovirus) is the FG human pancreatic adenocarcinoma cell line. This cell line is a fast growing variant of COLO357 that exhibits metastatic potential in mice. Compared to other tumor cell lines, FG cells are relatively resistant to the effects of AdN5 or Adp53 infection. FG cells were infected with AdN5, Adp53, or a combination of AdN5 and Adp53 (1:1) each at a total MOI of 50. Infection with an adenovirus designed to express green fluorescent protein (AdGFP) served as a control. Infection with AdN5 or Adp53 modestly decreased the growth rate of FG cells relative to treatment with AdGFP or mock infection (Fig. 1A). Infection with a combination of AdN5 and Adp53 had a greater inhibitory effect on FG cell growth than treatment with either adenovirus alone, all at the same total viral dose. Hence, the effects of combination gene therapy were greater than additive.

Forced expression of Thoc1 or p53 are known to induce apoptotic cell death in FG cells (15). To test whether the increased effects of combination gene therapy was due to increased apoptotic cell death, FG cells were infected with AdN5, Adp53 and AdN5+Adp53 for $72 \mathrm{~h}$ and the percentage 


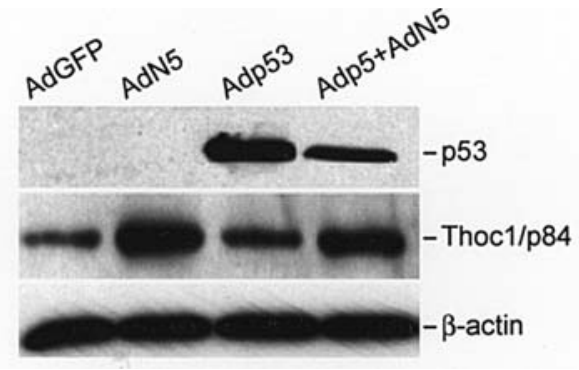

Figure 2. Thoc1/p84 and p53 expression levels in infected FG cells. FG cells were infected with indicated adenovirus at an MOI of 50 for $48 \mathrm{~h}$. The level of Thoc $1 / \mathrm{p} 84$ and p53 protein expression was determined by Western blotting. Identical blots were also probed with anti- $\beta$-actin antibody to control for protein loading. The positions of Thoc $1 / \mathrm{p} 84$, p53 and $\beta$-actin are indicated.

of apoptotic cells was measured by Annexin $\mathrm{V}$ binding and flow cytometry. A replication defective recombinant adenovirus expressing no exogenous protein (AdE1) served as a negative control. While either AdN5 or Adp53 treatment as single agents increased apoptosis relative to the control virus, combination adenoviral infection increased the percentage of apoptotic cells by a significant margin over single virus infections (Fig. 1B). Hence, the effects on tumor cell growth correlated with increases in apoptosis, suggesting that combination gene therapy inhibits FG cell growth by inducing apoptotic cell death.

We analyzed infected FG cells for Thoc1/p84 and p53 by Western blotting to assess gene transfer and protein expression (Fig. 2). After 48-h infection, p53 was not detected in AdGFP- or AdN5-infected FG cells but was observed in Adp53-infected cells and in AdN5+Adp53-infected cells. The level of p53 protein expression was 2-fold higher in Adp53-infected $(\mathrm{MOI}=50)$ cells compared to AdN5+Adp53infected $(\mathrm{MOI}=25+25)$ cells due to the lower dose of Ad53 in the combination treatment. An increase in Thoc1/p84 expression was observed in AdN5-infected FG cells or AdN5+Adp53-infected cells relative to AdGFP-infected cells, and again the level of increase was less in the combination treatment due to the lower relative dose of AdN5. These results indicated that growth inhibition correlated with increased p84N5 and p53 protein and indicated that the combination treatment had a greater effect on cell growth despite achieving lower levels of protein overexpression compared to the single agent treatments.

In order to test the potential utility of combination Thoc1/ p84 and p53 gene therapy in the treatment of pancreatic cancer, we tested the effects of AdN5 and Adp53 treatment in subcutaneous xenograft mouse model using FG cells. Viable cells were subcutaneously injected into the flanks of nude mice and tumors were allowed to grow for 3 weeks until they reached a palpable size. The tumors were then treated with three weekly intratumoral injections of AdN5, Adp53, AdGFP or AdN5+Adp53. Tumor volume was monitored until animals became moribund. The size of FG tumors was reduced upon treatment with AdN5 $(\mathrm{P}=0.075)$ relative to tumors treated with AdGFP (Fig. 3). Although Adp53 treatment slowed the growth of FG tumors relative to AdGFP-treated tumors, the difference was not statistically significant $(\mathrm{P}=0.47)$. Importantly,

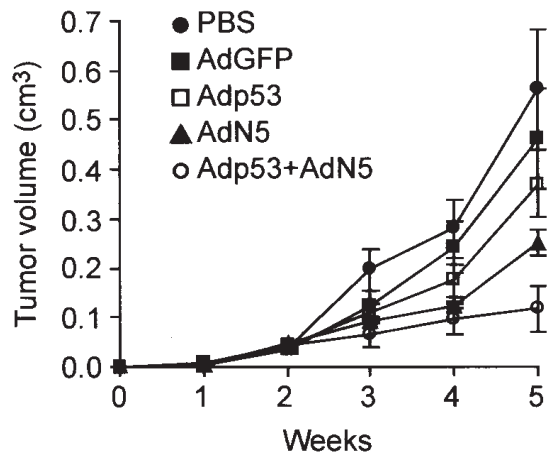

Figure 3. Effects of intratumoral injection with AdN5 and Adp53 on pancreatic tumor cell growth in vivo. Equal numbers of FG human pancreatic adenocarcinoma cells were subcutaneously implanted in nude mice. Three weeks later, palpable tumors were treated by intratumoral injection with the indicated virus. The same total viral dose was used in each case. Tumor volumes were measured weekly after implantation. Each data point represents the mean and standard deviation of the tumor volumes for at least six mice for each virus

AdN5+Adp53 treatment significantly inhibited tumor growth relative to AdGFP treatment $(\mathrm{P}=0.01)$ and combined treatment was better than either Adp53 $(\mathrm{P}=0.01)$ or AdN5 $(\mathrm{P}=0.04)$ treatment alone at the same total dose of virus. Hence, combination gene therapy had a greater than additive effect on reducing tumor growth relative to gene therapy with a single agent.

Thoc1/p84 was previously demonstrated to interact with the retinoblastoma tumor suppressor protein $(R b l)$. We tested whether combination Thoc1/p84 and Rb1 gene therapy may also cause a greater reduction in tumor cell growth compared to single agents. Pancreatic adenocarcinoma cells usually express $R b l$ protein, but this protein is deregulated by hyperphosphorylation due to the loss of cyclin dependent kinase inhibitor p16Ink4A expression that is common in pancreatic cancer (23). To overcome this limitation, we have used a recombinant adenovirus designed to express $R b 1$ protein with the serine residues at codons 608 and 612 replaced by alanines (AdRb). These substitutions render the mutant $R b 1$ protein resistant to hyperphosphorylation. To test the effect of a combination of both AdN5 and AdRb on the growth of cancer cells, FG cells were infected with AdGFP, AdN5, AdRb, or a combination of AdN5 and AdRb (1:1) at the MOI of 50. Infection with AdN5 or AdRb decreased the growth rate of FG cells relative to treatment with AdGFP or PBS. Infection with a combination of AdN5 and AdRb had an even greater effect on inhibition of FG cell growth than treatment with either adenovirus alone (Fig. 4A). As with p53 and Thoc1/p84 combination gene therapy, the extent of apoptosis induced by $\mathrm{AdRb}+\mathrm{AdN} 5$ was greater than either AdRb or AdN5 alone, at the same total viral dose (Fig. 4B).

In order to test the potential utility of the Thoc $1 / \mathrm{p} 84$ and $R b 1$ gene therapy combination in the treatment of pancreatic cancer, we tested the effects of AdN5 and AdRb alone or in combination using the subcutaneous FG cell xenograft mouse model. Using the same treatment schedule outlined above, treatment with the virus combination inhibited tumor growth to a greater extent than treatment with either virus alone at the same total viral dose (Fig. 5). 
A

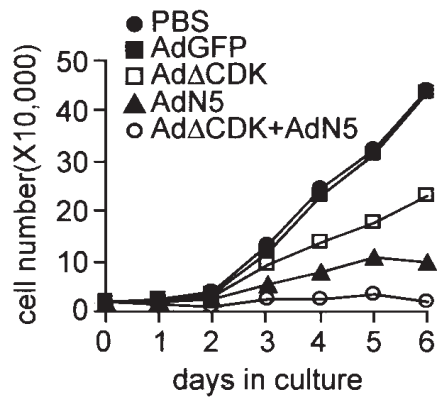

B

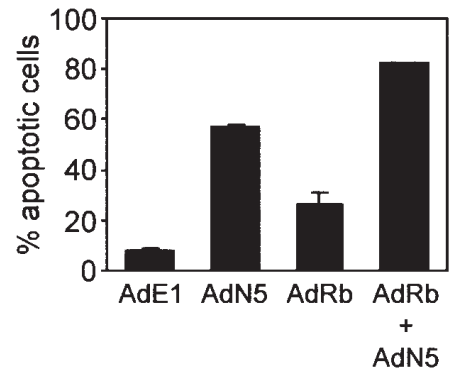

Figure 4. Effects of combination Thoc1/p84 and Rb1 gene therapy on pancreatic cancer cells in vitro. A, an equal number of FG cells were plated and treated at an MOI of 50 with the indicated virus or PBS. The total MOI of the combination of AdN5 and AdRb (1:1) was 50. Aliquots of the infected cells were harvested at the indicated times and the number of cells excluding trypan blue were counted using a hemacytometer. The data presented are the mean of three infections. The standard deviations from the mean are smaller than the symbols representing the data points. B, FG cells were infected with the indicated adenovirus at an MOI of 50 and harvested $72 \mathrm{~h}$ later. The percentage of apoptotic cells was measured using Annexin V staining and flow cytometry. The data represent the mean of three independent experiments.

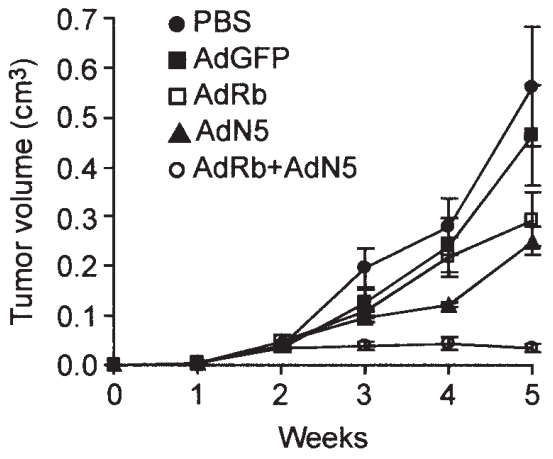

Figure 5. Effects of intratumoral injection with AdN5 and AdRb on pancreatic tumor cell growth in vivo. Equal numbers of FG human pancreatic adenocarcinoma cells were subcutaneously implanted in nude mice. Three weeks later, palpable tumors were treated by intratumoral injection with the indicated virus. The same total viral dose was used in each case. Tumor volumes were measured weekly after implantation. Each data point represents the mean and standard deviation of the tumor volumes for at least six mice for each virus.

\section{Discussion}

A number of potentially promising targets for the gene therapy of cancer have been identified in preclinical studies over the last several years. Particular attention has been focused on tumor suppressor genes, particularly those that trigger or sensitize tumor cells to apoptotic cell death. While these targets, like p53, have been effective in preclinical in vitro and animal studies, their effectiveness in clinical trials has been modest. The problems encountered in clinical trials undoubtedly involve technical limitations in the current ability to efficiently deliver the genes to the tumor. However, it is also becoming increasingly apparent that a substantial fraction of tumors are resistant to the effects of the transferred gene. By analogy to experience with cancer chemotherapeutics, therapeutic resistance may be overcome by simultaneous targeting of more than one distinct molecular pathway.

In the current study we test this possibility in a xenograft mouse model of human pancreatic adenocarcinoma that is resistant to the effects of p53 gene therapy. We examine the effects of three different genes that target distinct but possibly overlapping pathways. The p53 gene is important for mediating cellular responses to stress and when activated can trigger apoptotic cell death; tumor cells lacking p53 respond poorly to genotoxic stresses introduced by many commonly used chemotherapies. Thoc1/p84 plays a role in coupling transcriptional elongation with RNA processing. Deregulation of this protein sensitizes human tumor cells to the effects of DNA damage (17) and can trigger apoptotic cell death independently from p53 $(15,20)$. $R b 1$ is a tumor suppressor gene that restrains the cell cycle and may also have effects on cellular differentiation and survival. We find that treatment with a combination of Adp53+AdN5 or AdRb+AdN5 has a greater effect in inhibiting tumor cell growth in the p53resistant model of pancreatic cancer than either agent alone. Since the treatments are all done at the same total viral dose, the single agent treatments achieve a greater level of protein overexpression for the relevant protein than when used as part of a combination. These observations suggest that the effectiveness of the combination is greater than additive. Presumably modest overexpression of two genes that function in distinct pathways that are biologically relevant to tumor cell growth is more effective than significant overexpression of a single gene. These data support the use of gene therapy that targets both the p53 and Thoc1/p84 pathways, or the Thoc1/p84 and Rbl pathways, in tumors that are resistant to perturbation of the p53 pathway alone.

\section{Acknowledgements}

We thank Dr T.J. Liu (M.D. Anderson Cancer Center) for the kind gift of the $R b 1$ expressing recombinant adenovirus. This work was supported by grants to D.W.G. from the Wilson Foundation and the National Institutes of Health.

\section{References}

1. Kanduc D, Mittelman A, Serpico R, Sinigaglia E, Sinha AA, Natale C, Santacroce R, Di Corcia MG, Lucchese A, Dini L, Pani P, Santacroce S, Simone S, Bucci R and Farber E: Cell death: apoptosis versus necrosis. Int J Oncol 21: 165-170, 2002.

2. Leist $\mathrm{M}$ and Jaattela M: Four deaths and a funeral: from caspases to alternative mechanisms. Nat Rev Mol Cell Biol 2: 589-598, 2001.

3. Reed JC: Apoptosis-based therapies. Nat Rev Drug Discov 1: 111-121, 2002.

4. Favrot M, Coll JL, Louis N and Negoescu A: Cell death and cancer: replacement of apoptotic genes and inactivation of death suppressor genes in therapy. Gene Ther 5: 728-739, 1998.

5. Tseng JF and Mulligan RC: Gene therapy for pancreatic cancer. Surg Oncol Clin N Am 11: 537-569, 2002. 
6. Halloran CM, Ghaneh P, Neoptolemos JP and Costello E: Gene therapy for pancreatic cancer - current and prospective strategies. Surg Oncol 9: 181-191, 2000.

7. Bouvet M, Bold RJ, Lee J, Evans DB, Abbruzzese JL, Chiao PJ, McConkey DJ, Chandra J, Chada S, Fang B and Roth JA: Adenovirus-mediated wild-type p53 tumor suppressor gene therapy induces apoptosis and suppresses growth of human pancreatic cancer. Ann Surg Oncol 5: 681-688, 1998.

8. Ghaneh P, Greenhalf W, Humphreys M, Wilson D, Zumstein L, Lemoine NR and Neoptolemos JP: Adenovirus-mediated transfer of p53 and p16(INK4a) results in pancreatic cancer regression in vitro and in vivo. Gene Ther 8: 199-208, 2001.

9. Kimura M, Tagawa M, Takenaga K, Yamaguchi T, Saisho H, Nakagawara A and Sakiyama S: Inability to induce the alteration of tumorigenicity and chemosensitivity of p53-null human pancreatic carcinoma cells after the transduction of wild-type p53 gene. Anticancer Res 17: 879-883, 1997.

10. Vecil GG and Lang FF: Clinical trials of adenoviruses in brain tumors: a review of Ad-p53 and oncolytic adenoviruses. J Neurooncol 65: 237-246, 2003.

11. Zeimet AG and Marth C: Why did p53 gene therapy fail in ovarian cancer? Lancet Oncol 4: 415-422, 2003.

12. Durfee T, Mancini MA, Jones D, Elledge SJ and Lee W-H: The amino-terminal region of the retinoblastoma gene product binds a novel nuclear matrix protein that co-localizes to centers for RNA processing. J Cell Biol 127: 609-622, 1994.

13. Feinstein E, Kimchi A, Wallach D, Boldin M and Varfolomeev E: The death domain: a module shared by proteins with diverse cellular functions. Trends Biochem Sci 20: 342-344, 1995.

14. Evans RL, Poe BS and Goodrich DW: Nuclear localization is required for induction of apoptotic cell death by the $\mathrm{Rb}$-associated p84N5 death domain protein. Oncogene 21: 4691-4695, 2002.
15. Yin S, Bailiang W, Xie K and Goodrich DW: Adenovirusmediated N5 gene transfer inhibits tumor growth and metastasis of human carcinoma in nude mice. Cancer Gene Ther 9: 665-672, 2002.

16. Strasser K, Masuda S, Mason P, Pfannstiel J, Oppizzi M, Rodriguez-Navarro S, Rondon AG, Aguilera A, Struhl K, Reed R and Hurt E: TREX is a conserved complex coupling transcription with messenger RNA export. Nature 417: 304-308, 2002.

17. Li Y, Wang X, Zhang X and Goodrich DW: Human hHpr1/ p84/Thoc1 regulates transcriptional elongation and physically links RNA polymerase II and RNA processing factors. Mol Cell Biol 25: 4023-4033, 2005.

18. Rehwinkel J, Herold A, Gari K, Kocher T, Rode M, Ciccarelli FL, Wilm M and Izaurralde E: Genome-wide analysis of mRNAs regulated by the THO complex in Drosophila melanogaster. Nat Struct Mol Biol 11: 558-566, 2004.

19. Guo S, Hakimi MA, Baillat D, Chen X, Farber MJ, KleinSzanto AJP, Cooch NS, Godwin AK and Shiekhattar R: Linking transcriptional elongation and messenger RNA export to metastatic breast cancers. Cancer Res 65: 3011-3016, 2005.

20. Yin S, Hung MC and Goodrich DW: Adenovirus-mediated N5 gene transfer inhibits tumor cell proliferation by induction of apoptosis. Cancer Gene Ther 7: 985-990, 2000.

21. Doostzadeh-Cizeron J, Yin S and Goodrich DW: Apoptosis induced by the nuclear death domain protein p $84 \mathrm{~N} 5$ is associated with caspase-6 and NF-kappaB activation. J Biol Chem 275: 25336-25341, 2000.

22. Doostzadeh-Cizeron J, Terry NHA and Goodrich DW: The nuclear death domain protein p84N5 activates a $\mathrm{G}_{2} / \mathrm{M}$ cell cycle checkpoint prior to the onset of apoptosis. J Biol Chem 276: 1127-1132, 2001.

23. Schneider G and Schmid RM: Genetic alterations in pancreatic carcinoma. Mol Cancer 2: 15, 2003. 